\title{
Characterisation of Transient Behaviours in Rotordynamic Vibrations from Experimental Data Using Harmonic Wavelets
}

\author{
Valeta Carol Chancey \\ Duke University, Durham, North Carolina, USA \\ George T. Flowers ${ }^{\dagger}$ and Candice L. Howard \\ Auburn University, Auburn, Alabama, USA
}

(Received 7 August 2000; accepted 18 September 2000)

\begin{abstract}
Wavelets are versatile tools for the analysis of vibration signals. Wavelet-based methods are especially valuable for the analysis of vibrations that are not steady state. However, it is sometimes difficult to extract physically relevant patterns from the great wealth of information contained in the wavelet decomposition of a vibration signal. By examining the absolute values of harmonic wavelet coefficients, the application of harmonic wavelets to the vibration analysis of rotating machinery is explored. The basics of the strategy are explained. Example experimental data collected from a bench-top test rig with a journal bearing exhibiting oil whirl was analysed to illustrate the proposed methods.
\end{abstract}

${ }^{\dagger}$ Member of the International Institute of Acoustics and Vibration (IIAV)

\section{NOMENCLATURE}

$\omega \quad$ - rotor speed or frequency, $\mathrm{rad} / \mathrm{s}$

$\phi \quad-$ phase angle, rad

$t \quad$ - time, $\mathrm{s}$

$i \quad-$ an integer subscript

$f_{i} \quad$ - discrete values of the input signal

$F_{i} \quad-$ semi-coefficient output from the FFT

$M \quad$ - number of components of $f(t)$ when a signal is described as $f(t)=\sum_{i=1}^{M} g_{i}(t) \cos \left(\omega_{i} t-\phi_{i}\right)$

$N \quad$ - integer length of signal or number of data points, usually $N=2^{n}$

$n \quad$ - integer exponent of 2 used to express length of signal

$f(t)$ - function representing data or signal

$g(t) \quad$ - growth function

$w(t) \quad$ - harmonic wavelet functional form

$W(\omega)$ - Fourier transform of the harmonic wavelet

$a_{j, k} \quad$ - wavelet coefficient at level $j$ translated by $k$, used as $a_{k}$ for the harmonic wavelet coefficient within a given level

$j \quad$ - the integer indicating the level of wavelet decomposition, also $\sqrt{-1}$ in complex number representation

$m, n$ - the combination represents the beginning and end of a particular level in the use of a form of the harmonic wavelet transform, a new level where the original harmonic wavelet family is redefined

AHWC - absolute harmonic wavelet coefficient

FFT - fast Fourier fransform

HW - harmonic wavelet

IFFT - inverse fast Fourier transform

\section{INTRODUCTION}

The field of machinery diagnostics is a dynamic and active field for engineering research in part because of continued development and the growing dependence upon costly equipment. In particular, breakdowns of high speed rotating machinery can be extremely costly and occur with no apparent warning. As new technology has become readily available, the diagnostic capabilities possible have evolved. Today, infrared measurements, oil testing, and vibration analyses are all components of an effective proactive strategy for machinery developmental testing and maintenance. Of the three, vibration analysis is usually the key element of any successful program. Three basic situations exist where it may be of value: (1) diagnosis of machinery problems during design, (2) quality assurance at the installation stage, and (3) regular health monitoring during normal operation. ${ }^{1}$ In these cases, vibration measurements are taken to help understand the conditions or operation of complex systems. Current analysis methods and representations, based upon Fourier and time series techniques that have been available for several years, may not always provide adequate indications of machinery defects.

Often it is the role of vibration analysis to aid in the identification of specific machinery problems. For certain phenomena, it may be difficult or impossible to distinguish between possible causes based solely upon current vibration analysis techniques. For example, there may be no visible distinction between a self-excited instability limited by some non-linear effect and forced responses resulting from the surging of the working fluid in the machine. The vibration signals may be of similar frequency and amplitude with the associated time traces and Fourier diagrams also extremely similar. The beginning of a solution to this problem, involv- 